\title{
Medical Abortion: Outcomes in a Family Medicine Setting
}

\author{
Linda Prine, MD, Caitlin Shannon, JD, MPH, Ginger Gillespie, MD, \\ William A. Crowden, Jennifer Fortin, MPH, Michelle Howe, PA-C, and \\ Ilana Dzuba, MHS
}

Purpose: To compare outcomes of early medical abortion with mifepristone and misoprostol in a family medicine setting and specialized reproductive health clinics.

Methods: This study used data collected from a prospective, open-label, randomized trial of oral versus buccal misoprostol efficacy. A secondary analysis was performed, evaluating efficacy, acceptability, and interventions after medication at the family medicine site compared with the 6 specialized reproductive health sites.

Results: Comparing data from patients in the family medicine setting $(\mathrm{n}=116)$ to specialized reproductive health sites $(n=731)$ revealed no difference in overall efficacy $(95.7 \%$ vs $93.4 \% ; P=.351)$. The family medicine site used a second dose of misoprostol more frequently than the other sites $(6.9 \%$ vs $2.5 \% ; P=.018)$. In addition, uterine aspiration after medical abortion at the family medicine site was not used for "medically necessary" reasons whereas reproductive health clinics used it $2.6 \%$ of the time (marginally significant; $P=.094$ ). Patient satisfaction at family medicine sites was comparable to the other sites $(91.2 \%$ vs $92.0 \% ; P=.792)$.

Conclusion: Medical abortion has similar efficacy and patient satisfaction when offered in a family medicine practice or at a reproductive health specialty clinic. These findings should reassure family physicians that medical abortion can be offered safely in their practices. (J Am Board Fam Med 2010;23: 509-513.)

Keywords: Practice-based Research, PBRN, Abortion, Misoprostol, Family Medicine

The United States has a major shortage of physicians who provide abortions, and the number of providers continues to decline each year. ${ }^{1}$ Despite the need for more providers, many barriers have prevented family physicians specifically from providing abortion services to their patients. Some liability insurance carriers have refused to cover

This article was externally peer reviewed.

Submitted 28 September 2009; revised 5 February 2010; accepted 15 February 2010.

From the Institute for Family Health (LP, GG) and Gynuity Health Projects (CS, ID), New York, NY; Planned Parenthood of Waco, TX (WAC); Planned Parenthood League of Massachusetts, Boston (JF); and the Family Planning Associates Medical Group, Chicago, IL (MH).

Funding: Funding was provided by Gynuity Health Projects.

Prior Presentation: Presented as a poster at the Society for Teachers of Family Medicine Northeast Regional Meeting, October 2007, Pittsburgh, PA; the Annual Meeting of the National Abortion Federation, April 2008; and at the North American Primary Care Research Group Annual Meeting, November 2008, San Jose, Puerto Rico.

Conflict of interest: none declared.

Corresponding author: Linda Prine, MD, 16 East 16th St,

New York, NY 10011 (E-mail: lindaprine@earthlink.net). family physicians for abortion procedures, although there is no reason to expect any change in risk when the medication is provided by a family physician. ${ }^{2}$ In addition, health insurance companies have denied claims for reimbursement to family physicians. $^{3}$ Finally, most family medicine residency training programs fail to include abortion care in the curriculum, and the Residency Review Committee does not address abortion skills for family medicine residents in its guidelines. ${ }^{4}$

Family medicine sites have been involved in medical abortion research in the United States since the outset and several published studies have demonstrated good outcomes for medical abortion performed in family medicine settings. ${ }^{5-8}$ (In patient education materials, we used the wording "medication abortion" to make it clear to patients that the abortion is caused by a pill; however, the convention used in the medical literature has been to call it "medical abortion," so to be consistent with the published literature we have used that term here.) No published studies have compared 
medical abortion outcomes in the family medicine setting versus outcomes in specialized reproductive health clinics. This study was conducted from September 2006 until August 2007 to examine the efficacy of buccal and oral administration of $800 \mu \mathrm{g}$ misoprostol during the 63 days since the last menstrual period; the primary analysis on comparative efficacy has been published previously. ${ }^{9}$ Using a secondary data analysis, this article examines the outcomes and patient satisfaction of medical abortion performed in the family medicine setting compared with those performed at specialized reproductive health sites.

\section{Methods}

We used data collected during a prospective, multicenter, open-label, randomized trial that was designed to compare the efficacy of oral and buccal administration of $800 \mu \mathrm{g}$ of misoprostol after mifepristone $200 \mathrm{mg}$ during the 63 days since the last menstrual period. Seven institutions participated in the study: one family medicine practice at 2 locations and 6 specialty reproductive health sites. The family medicine practice was part of the Family Medicine Research Network, a practice-based research network.

The family medicine setting was an urban residency program practice in lower Manhattan, NY, where the women were cared for by faculty and residents, many of whom were the patients' regular physicians. In addition, some patients were referred to the family medicine site specifically for medical abortions. The specialized reproductive health sites mainly had advanced practice clinicians providing medical abortions. The complete methods and primary data analysis for this study have been previously published. ${ }^{9}$

Patients eligible for medical abortion and pregnant at a gestational age $<64$ days (as defined by ultrasound and/or by last menstrual period and confirmed by physical examination) were recruited for the study. Participating women gave informed consent, were given mifepristone, and were then randomized to either oral or buccal misoprostol. Before leaving the clinic, all women were given instructions for administering misoprostol at home 24 to 36 hours later. Follow-up visits were scheduled in 1 to 2 weeks to assess abortion status and identify potential complications, such as excessive bleeding or retention of a nonviable pregnancy.
Successful medical abortion was defined as a complete abortion without a uterine aspiration procedure at any point, regardless of the number of doses of misoprostol that were taken. Abortion completion was assessed using clinical history, ultrasound findings, and/or serial $\beta$-human chorionic gonadotropin levels. Intervention with uterine aspiration performed at the discretion of the provider-for ongoing pregnancy, for persistent gestational sac, or at the patient's request-was documented. During the follow-up visit, the patient's home diary was collected, which documented side effects and bleeding, and an exit interview was administered, which assessed side effects, satisfaction with the experience, and acceptability of the medical abortion method. Adherence to the medical abortion regimen was determined according to the information provided by the women during the follow-up visit(s). Participants were considered lost to follow-up if there was no physical or telephone contact with them by the end of the study to allow for a final outcome determination.

Between September 2006 and May 2007, 966 participants were enrolled; follow-up of participants continued through August 2007. At the family medicine site, 138 women were enrolled, 116 of whom had analyzable outcome data; at the remaining sites 828 patients were enrolled, 731 of whom had analyzable outcome data. Those who were not included withdrew from the study, were lost to follow-up, or had protocol violations. Adherence to the protocol was determined according to information provided during the follow-up visit. Data were analyzed using SPSS software (version 15.0; SPSS, Inc., Chicago, IL) and are presented based on a per protocol analysis, restricted to participants who administered misoprostol as prescribed by the protocol.

This article reports on a secondary analysis comparing the family medicine site with the specialty reproductive health sites in terms of patient satisfaction, overall outcomes, and rates of intervention after medication was taken. For all analyses, $P \leq$ .05 was considered statistically significant. $P$ was measured using an analysis of variance or MannWhitney test for continuous variables and with a $\chi^{2}$ test or Fischer's exact test, as appropriate, for frequencies and categorical variables. Analysis adjusting for educational status, gestational age, and gravidity were conducted using a logistic regression model; the route of administration was controlled 
Table 1. Participant Characteristics by Clinic Type

\begin{tabular}{lcc}
\hline & $\begin{array}{c}\text { Family } \\
\text { Medicine } \\
(\mathrm{n}=138)\end{array}$ & $\begin{array}{c}\text { Specialty } \\
\text { Reproductive } \\
\text { Health } \\
(\mathrm{n}=828)\end{array}$ \\
\hline Characteristic & $26.6(6.5)$ & $26.1(5.8)$ \\
Age (mean years [SD]) & & \\
Educational level & $13(9.4)$ & $61(7.3)$ \\
$<$ High school & $49(35.5)$ & $461(55.7)$ \\
High school graduate* & $60(43.5)$ & $248(30.0)$ \\
University graduate* & $13(9.4)$ & $38(4.6)$ \\
Postgraduate degree* & $3(2.2)$ & $20(2.4)$ \\
$\quad$ Unknown & $67(48.6)$ & $403(48.7)$ \\
Previous abortions & $2(1-4)$ & $3(1-4)$ \\
Gravidity* (median [Q1-Q3]) & $48.3(9.2)$ & $50.1(8.1)$ \\
Gestational age* (days [SD]) & $13(9.4)$ & $79(9.5)$ \\
Lost to follow-up & &
\end{tabular}

Values provided as $\mathrm{n}(\%)$ unless otherwise indicated. ${ }^{*} P<.05$.

for by study group randomization, not by logistic regression.

\section{Results}

Women enrolled at family medicine and specialty reproductive health sites were similar with respect to age, marital status, and previous abortions (Table 1). More family medicine patients had more than a high school education, compared with the specialty reproductive health clinic patients (risk ratio, $0.72 ; 95 \% \mathrm{CI}, 0.59-0.87)$. In addition, women who were enrolled in the family medicine site had lower median gravidity than the women who were seem at the specialty reproductive health sites ( 2 vs $3 ; P=.003$ ) and had a slightly lower mean gestational age (48.3 vs 50.1 days; $P=.018$ ).

There was no difference in medical abortion efficacy between the family medicine and the spe- cialty reproductive health sites $(95.7 \%$ vs $93.4 \%$, respectively; $P=.351$ ) (Table 2 ). Controlling for gestational age, gravidity, and education, the comparison remained statistically insignificant (crude versus adjusted analysis: risk ratio, $1.56[95 \% \mathrm{CI}$, 0.61-4.01] vs risk ratio, 1.39 [95\% CI, 0.53-3.65], respectively). If an incomplete abortion (defined as evidence that not all of the products of conception had been totally expelled) was diagnosed during the follow-up visit, providers at the family medicine site more frequently used a repeat dose of misoprostol than did those at the specialty reproductive health sites $(6.9 \%$ vs $2.5 \% ; P=.018)$. Intervention with a uterine aspiration procedure was used to manage continuing pregnancy, retained gestational sac, or was performed at the patient's request. The rates of these interventions were not statistically different between the 2 types of sites. In contrast, 19 aspirations (2.6\%) for medical reasons other than incomplete abortion, including prolonged nausea and vomiting or persistent bleeding, were performed occasionally in the specialty reproductive health sites, compared with none at the family medicine site. The lower intervention rate at the family medicine site was only marginally significant $(P=.094)$. However, although these reasons were categorized as "medically necessary" for data collection, they are not definitive indications for surgical intervention but rather are based on a physician's judgment.

Twenty-six women $(3.0 \%)$ reported visits to an emergency department during the study period primarily for pain and bleeding, but also for fever, nausea, fainting, and a presumed allergic reaction; 5 patients $(4.3 \%)$ were from the family medicine site and 21 patients $(2.9 \%)$ were from the specialty reproductive health sites. The majority of these

Table 2. Medical Abortion Outcome by Clinic Type

\begin{tabular}{|c|c|c|c|c|}
\hline Outcome & $\begin{array}{l}\text { Family Medicine } \\
(\mathrm{n}=116)(\mathrm{n}[\%])\end{array}$ & $\begin{array}{l}\text { Specialty Reproductive Health } \\
(\mathrm{n}=731)(\mathrm{n}[\%])\end{array}$ & $P$ & Risk Ratio $(95 \% \mathrm{CI})$ \\
\hline Success & $111(95.7)$ & $683(93.4)$ & .351 & $1.02(0.98-1.07)$ \\
\hline \multicolumn{5}{|l|}{ Reason for surgical intervention } \\
\hline Ongoing pregnancy & $2(1.7)$ & $17(2.3)$ & $1.000^{*}$ & $0.74(0.17-3.17)$ \\
\hline Persistent sac & $2(1.2)$ & $12(1.6)$ & $1.000^{*}$ & $1.05(0.24-4.63)$ \\
\hline Medically necessary & $0(0.0)$ & $19(2.6)$ & $.094^{*}$ & N/A \\
\hline Patient request & $1(0.9)$ & $0(0.0)$ & $.137^{*}$ & N/A \\
\hline Administered second dose of misoprostol & $8(6.9)$ & $18(2.5)$ & .018 & $2.80(1.25-6.29)$ \\
\hline
\end{tabular}

*Fischer's 2-sided exact test. 


\begin{tabular}{lccc}
\hline & $\begin{array}{c}\text { Family Medicine } \\
(\mathrm{n}=116)\end{array}$ & $\begin{array}{c}\text { Specialty Reproductive Health } \\
(\mathrm{n}=731)\end{array}$ & $P$ \\
\hline Satisfaction with procedure & $104(91.2)$ & $663(92.0)$ & .792 \\
Procedure not/slightly difficult & $71(62.3)$ & $520(72.1)$ & .032 \\
Pain acceptable & $76(66.7)$ & $480(66.6)$ & .984 \\
Side effects acceptable & $87(76.3)$ & $530(73.5)$ & .526 \\
Time to completion acceptable & $92(80.7)$ & $601(83.4)$ & .483 \\
\hline
\end{tabular}

Values provided as $\mathrm{n}(\%)$.

*Satisfied means that women reported that the procedure was either very satisfactory or satisfactory. Acceptable means that women reported side effects, pain, or time of procedure as either acceptable or very acceptable.

patients $(80.8 \%)$ were not admitted. One participant from the family medicine site was hospitalized during the study period for ruptured ectopic pregnancy; 2 participants from the specialty reproductive health sites were hospitalized for pulmonary embolus and right hip pain, respectively. The reasons for all hospitalizations were determined to be unrelated to the study protocol.

More than $90 \%$ of participants were highly satisfied with the experience and the majority found the side effects, pain, and time to completion acceptable (Table 3). The same level of satisfaction with the process was found among women treated at the family medicine site and the specialty reproductive health sites $(92.0 \%$ vs $91.2 \%$, respectively; $P=.792)$. A higher percentage of women treated at the specialty reproductive health sites perceived the procedure as either not or only slightly difficult compared with those treated at the family medicine site $(72.1 \%$ vs $62.0 \%$, respectively; $P=.032)$. A logistic regression analysis of the perceived difficulty of the experience found a marginally significant relationship to education level, gravidity, and previous abortion (crude versus adjusted analysis: risk ratio, 0.599 [95\% CI, 0.396-0.906] vs risk ratio, 0.684 [95\% CI, 0.446-1.050]).

\section{Discussion}

This secondary analysis supports the integration of early medical abortion care into in the basket of services offered by family medicine practices. We found that the mifepristone-misoprostol regimens that were tested were highly effective in this setting and that the efficacy was comparable to that achieved in specialty reproductive health sites. Although the 2 patient cohorts were different with respect to education, gestational age, and gravidity, these factors did not significantly alter the clinical outcome. Moreover, our results confirm those reported in a previous study that demonstrated women treated in family medicine practices had high success rates with early medical abortion. In that study, among 236 women treated at 3 family medicine practices, the efficacy rate was $99.6 \%$, with only one aspiration procedure performed for an ongoing pregnancy through 63 days' gestation. ${ }^{3}$ In addition, a prospective observational study at 5 family medicine sites, including 4 residency practices, found a success rate of $96.5 \%$ for early medical abortion. ${ }^{8}$

There was one ruptured ectopic pregnancy in the family medicine group and one pulmonary embolus in the specialized clinic group. Both of these outcomes are secondary to pregnancy and should not be considered evidence that medical abortion is unsafe in either setting. A recent review of the published literature about medical abortion found that ectopic pregnancy went undetected in only 10 of every 44,789 women $(0.02 \%)$ undergoing medical abortion. ${ }^{10}$

When comparing the rate of interventions between the family medicine and specialty reproductive health sites, we found some practice differences. Physicians at the family medical site used additional doses of misoprostol more often than providers at the specialty reproductive health sites. It is possible that the surgically-trained practitioners at the reproductive health sites tended to recommend an aspiration procedure whereas family physicians were more prone toward management with misoprostol. However, this study was not powered to examine a difference in rates of procedural interventions by type of study site. Therefore, further research would be necessary to properly examine the impact of provider type and setting on rates of intervention. 
Acceptability and patient satisfaction rates were comparable between the clinic groups. Women attending specialty reproductive health sites, however, were more likely to report that the procedure was easier. This difference could be attributed in part to the greater median gravidity of women at the reproductive health sites. On average, women having more experience with pregnancy and childbirth may be more likely to find the cramping and bleeding of a medical abortion easier to manage. Educational attainment may also affect patients' perceptions of the ease of the process; in particular, it may create a different set of expectations. On average, the women at the family medical sites had greater education attainment and, because educational attainment was positively correlated with finding the process more difficult to manage, differences in educational attainment also may contribute to this difference. Because most urban family medicine residency practices have few patients with high education levels, this finding may not be generalizable to other family medicine residency sites; the findings may be more applicable to private family medicine practices. The efficacy and satisfaction level of patients from all backgrounds was high, the complication rate was low, and the degree to which the process was found to be difficult was generally low; therefore, the overall generalizability of our findings to other family medicine settings seems reasonable.

\section{Conclusion}

This article demonstrates that medical abortion is a highly effective and safe procedure when provided in a family medicine practice. Moreover, this procedure is simple to provide and requires few interventions beyond physical examination and the dispensation of medications. Concerns about complications and the need for "back-up" aspiration procedures are real for family physicians. Nonetheless, our data demonstrate that family physicians can manage complications of medical abortion and that women treated in family medicine practices had outcomes comparable to those who were treated at specialty reproductive health clinics. In addition, our data can be used by liability and reimbursement carriers to justify coverage of family physicians who provide medical abortion. Removing such barriers can expand access to this important service, particularly in rural areas where access to abortion providers is limited.

We would like to acknowledge the family medicine residents and nurses at Phillips Family Practice, as well as all the staff at the reproductive health sites who worked so hard on this study.

\section{References}

1. Jones RK, Zolna MRS, Henshaw SK, Finer LB. Abortion in the United States: incidence and access to services, 2005. Perspect Sex Reprod Health 2008;40:6-16.

2. Dehlendorf CE, Grumbach K. Medical liability insurance as a barrier to the provision of abortion services in family medicine. Am J Public Health 2008;98:1770-4.

3. Prine LW, Lesnewski R. Medication abortion and family physicians' scope of practice. J Am Board Fam Pract 2005;18:304-6.

4. Accreditation Council for Graduate Medical Education. Family medicine program requirements. Available at http://www.acgme.org/acWebsite/RRC_ 120/120_prIndex.asp. Accessed 15 June 2009.

5. Schaff EA, Fielding SL, Westhoff C. Randomized trial of oral versus vaginal misoprostol at one day after mifepristone for early medical abortion. Contraception 2001;64:81-5.

6. Schaff EA, Fielding SL, Eisinger SH, Stadalius LS, Fuller L. Low-dose mifepristone followed by vaginal misoprostol at 48 hours for abortion up to 63 days. Contraception 2000;61:41-6.

7. Prine L, Lesnewski R, Berley N, Gold M. Medical abortion in family practice: a case series. J Am Board Fam Pract 2003;16:290-5.

8. Bennett IM, Baylson M, Kalkstein K, Gillespie G, Bellamy SL, Fleischman J. Early abortion in family medicine: clinical outcomes. Ann Fam Med 2009;7: $527-33$.

9. Winikoff B, Dzuba IG, Creinin MD, et al. Two distinct oral routes of misoprostol in mifepristone medical abortion: a randomized controlled trial. Obstet Gynecol 2008;112:1303-10.

10. Shannon C, Brothers LP, Philip NM, Winikoff B. Ectopic pregnancy and medical abortion. Obstet Gynecol 2004;104:161-7. 